\title{
Outlier and Target Detection in Aerial Hyperspectral Imagery: A Comparison of Traditional and Percentage Occupancy Hit or Miss Transform Techniques
}

\author{
Andrew Young ${ }^{\mathrm{a}}$, Stephen Marshall ${ }^{\mathrm{a}}$, and Alison Gray ${ }^{\mathrm{b}}$

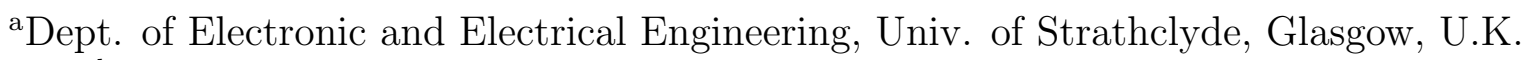 \\ ${ }^{\mathrm{b}}$ Dept. of Mathematics and Statistics, Univ. of Strathclyde, Glasgow, U.K.
}

\begin{abstract}
The use of aerial hyperspectral imagery for the purpose of remote sensing is a rapidly growing research area. Currently, targets are generally detected by looking for distinct spectral features of the objects under surveillance. For example, a camouflaged vehicle, deliberately designed to blend into background trees and grass in the visible spectrum, can be revealed using spectral features in the near-infrared spectrum. This work aims to develop improved target detection methods, using a two-stage approach, firstly by development of a physics-based atmospheric correction algorithm to convert radiance into reflectance hyperspectral image data and secondly by use of improved outlier detection techniques. In this paper the use of the Percentage Occupancy Hit or Miss Transform is explored to provide an automated method for target detection in aerial hyperspectral imagery.
\end{abstract}

Keywords: Hyperspectral Imaging, Remote Sensing, Sequential Maximum Angle Convex Cone (SMACC), Hit or Miss Transform, Percentage Occupancy Hit or Miss Transform, Outlier Detection, Target Detection

\section{INTRODUCTION}

In the area of remote sensing aerial hyperspectral imagery is a rapidly growing research area. ${ }^{1}$ The generally used technique for detecting targets is by using the distinct spectral features of the objects under surveillance to differentiate them from surrounding materials. For the case of a camouflaged vehicle, the vehicle is deliberately designed to blend into the background grass and trees in the visible spectrum. However by looking at spectral features in the near-infrared spectrum, such as the chlorophyll edge, the vehicle can be revealed. The received spectral radiance from a remote object depends on the spectral characteristics of the solar illumination and atmospheric attenuation at the location and time concerned. The prevailing atmospheric water vapor content in the ground to air path has a particularly strong effect on measured radiances in the near-infrared region.

The aim of this project is to develop a two stage target detection method that is an improvement over what is currently available. The first part of this two stage process is a physics-based atmospheric correction algorithm to convert the radiance hyperspectral image into reflectance. The second stage is looking at an improved target detection method.

In this paper we consider the second stage of this process. In the linear mixing model ${ }^{2}$ the radiance measured at each pixel is assumed to be the linear combination of the radiance of each material present in the pixel. The non-linear mixing model ${ }^{3}$ the radiance measured is assumed to be a weighted sum of the radiance of each material within the pixel plus the contribution due to scattering from both the sun to ground path and ground to sensor path. Here we investigate several outlier detection techniques used on the raw image data to find pure pixels, or outliers, those pixels that have not been mixed with the surrounding materials.The results are then compared with a validated technique, the Sequential Maximum Angle Convex Cone (SMACC). ${ }^{4}$

Andrew Young: E-mail: andrew.young.101@strath.ac.uk, Telephone: 01415487972 


\section{IMAGE ACQUISITION}

All the data used in this project was supplied by BAE Systems Advanced Technology Centre, the Defence Science and Technology Laboratory (DSTL) and the University Defence Research Collaboration (UDRC). The imagery is all aerial hyperspectral imagery acquired from an aeroplane flying at approximately $0.781 \mathrm{~km}$ and $1.197 \mathrm{~km}$ with a mounted hyperspectral sensor. Figure 1 shows two false-colour representations of these images $(636 \mathrm{~nm}, 555 \mathrm{~nm} 460 \mathrm{~nm}$ respectively for R, G, B).

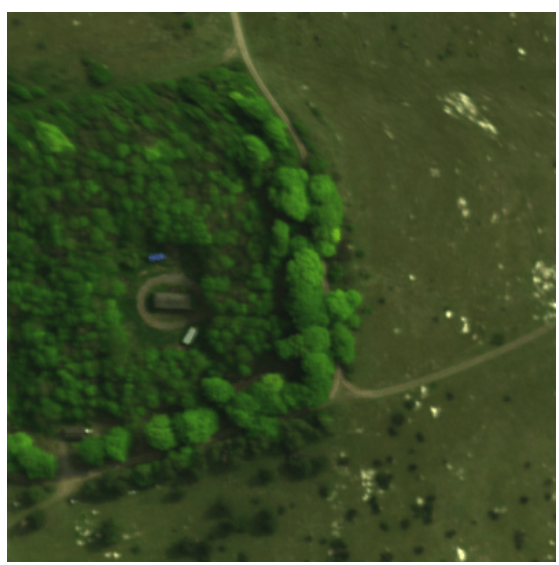

(a) 18th May 2014 at 11:29 in Moll Harris, Salisbury, Wiltshire

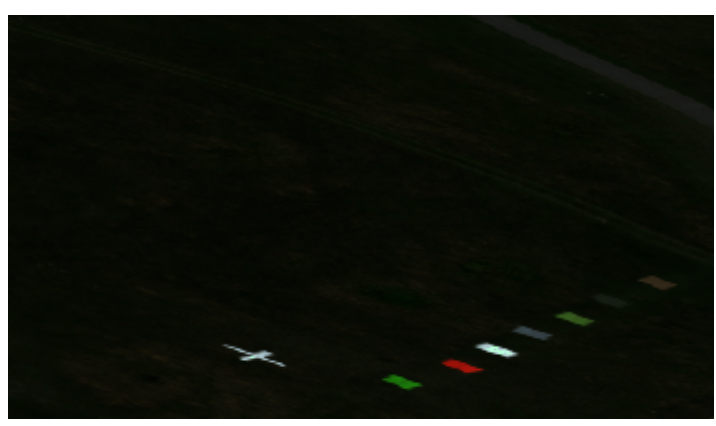

(b) 15th August 2014 at 15:17 in Porton Down, Salisbury, Wiltshire

Figure 1: False-colour representations of hyperspectral images

\section{TECHNIQUES}

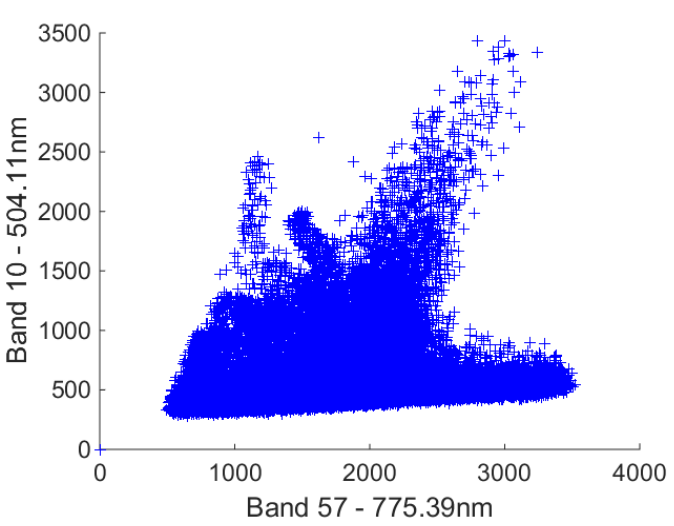

(a) Moll Harris data using bands 10 and 57

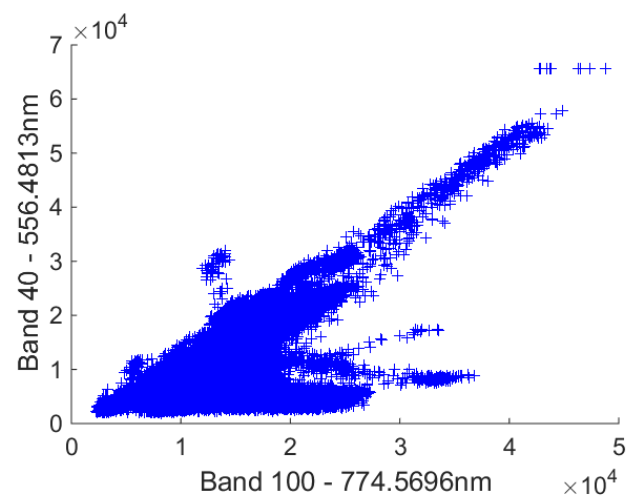

(b) Porton Down data using bands 40 and 100

Figure 2: Scatter plot for both hyperspectral images

Outliers are used in hyperspectral imagery to find materials and objects that are spectrally different from the image background; Figure 2 shows two example scatter plots created using two bands of each of the datasets. As both scenes are mostly trees and grass which are spectrally similar, there is a large cluster of pixels with similar radiance values in each band. The outliers or targets in the scene are the pixels further from this cluster and the centre of the data. Pixels closer to the centre are more mixed than more distant ones. By determining these outliers it is possible to detect unusual objects in the image that require further attention. Numerous techniques are used to find outliers in hyperspectral 
data. ${ }^{5}$ Here we consider two techniques described below, to try to improve upon the standard methods also described below.

\subsection{Sequential Maximum Angle Convex Cone (SMACC)}

1. Convert 3D hyperspectral image data cube into 2D matrix of pixel vectors (no. of pixels $\mathrm{x}$ no of bands) (Figure 3).

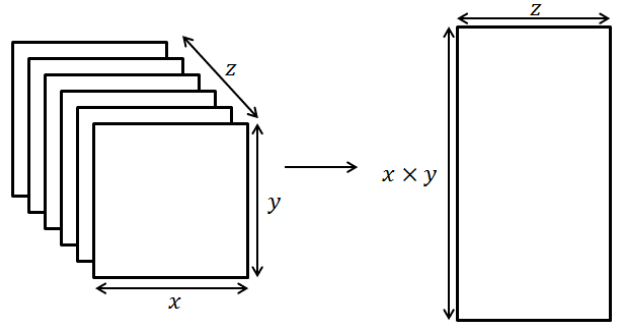

Figure 3: Conversion from 3D hyperspectral image to 2D matrix of pixel vectors

2. Calculate the squared length of each pixel vector, see Equation 1. The pixel with the maximum length first endmember (EM) is selected as the first EM. Store spectral profile of first EM.

$$
\text { pixel vector length }=\sqrt{\vec{v} \times \vec{v}^{T}}
$$

3. A subspace projection is then performed with each pixel vector, to calculate the abundance of that EM (pixel vector) in each pixel, see Figure 4, this forms an abundance image (AI) for EM one. Subtract abundance from image to produce updated data set.

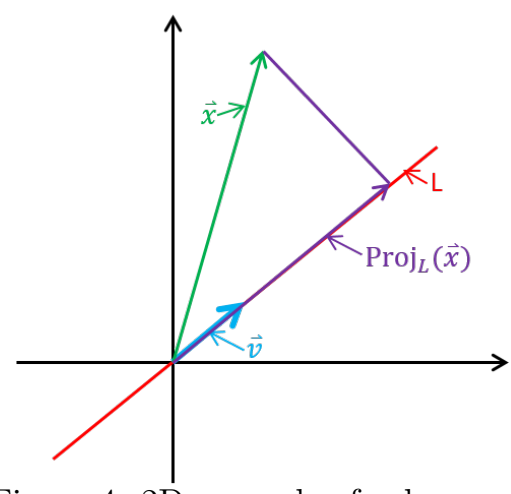

Figure 4: 2D example of subspace projection

4. The final step is then to remove all reference to this EM from the original dataset $\left(h_{0}\right)$; this is done by using Equation 2.

$$
h_{1}=h_{0}-E M \times A I
$$

5. Repeat steps 2-4 until required number of EMs are found or residual limit is reached. The residual is $\log _{10} \max (\text { length })^{2}$. 


\subsection{Mahalanobis Distance}

To calculate the Mahalanobis Distance ${ }^{6} \mathrm{MD}(\mathrm{x})$ in Equation 3 for an image pixel vector $\mathrm{x}$ requires first calculating a mean pixel vector $(\mu)$ containing the average pixel intensity values for all the bands or wavelengths measured, as well as an estimated covariance matrix $(\Gamma)$ of the pixel intensity values for all of the bands using Equation 4, where $\mathrm{N}$ is the number of pixels in the image. Doing this for every pixel vector gives a measure of how far that pixel is from the mean pixel vector. Larger values indicate more extreme pixel vectors.

$$
\begin{gathered}
M D(x)=\sqrt{(x-\hat{\mu})^{T} \Gamma^{-1}(x-\hat{\mu})} \\
\Gamma=\frac{1}{N} \sum_{n=1}^{N}(x-\hat{\mu})(x-\hat{\mu})^{T}
\end{gathered}
$$

To determine outliers, the maximum MD was found and any points with an MD larger than $25 \%$ of the maximum were considered to be outliers (Figure 5).

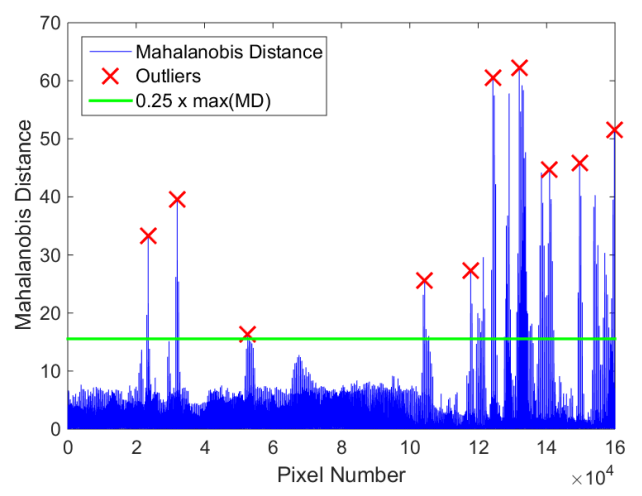

(a) Moll Harris data

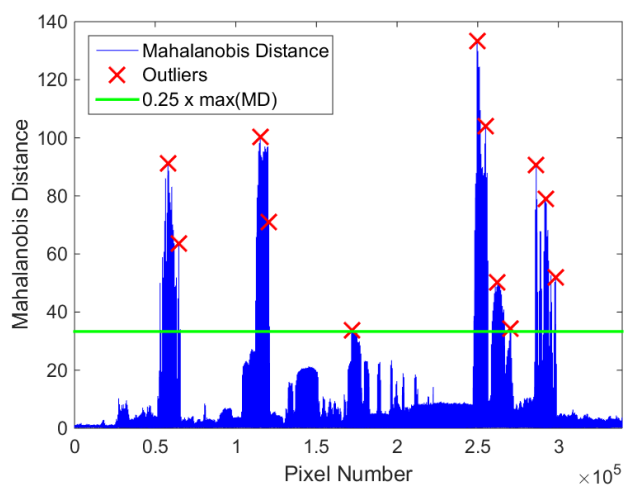

(b) Porton Down data

Figure 5: Mahalanobis Distance with all peaks highlighted. Green line shows the chosen threshold line.

\subsection{Percentage Occupancy Hit or Miss Transform}

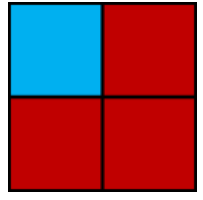

(a)

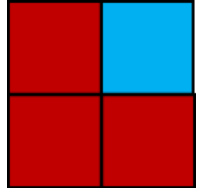

(b)

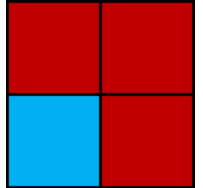

(c)

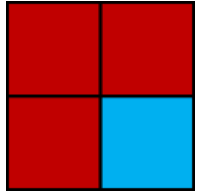

(d)

Figure 6: Four states used to detect outliers; blue is foreground, red is background

As the standard Hit or Miss Transform ${ }^{7}$ method does not allow for any target pixels to be in the background, the Percentage Occupancy Hit or Miss Transform (POHMT) ${ }^{8}$ is a more robust approach to detect outliers in the scatter plot. Figure 6 shows the four structuring elements used to implement this.

First the scatter plot was divided into several small cells, depending on the data and scatter plot chosen (Figure 7). The cells vary from 5x5 to 150x150 pixels in size. A new smaller image was then created. The number of pixels from the original image present in every cell of the scatter plot was counted and this value was taken as the corresponding pixel value in the new image. 


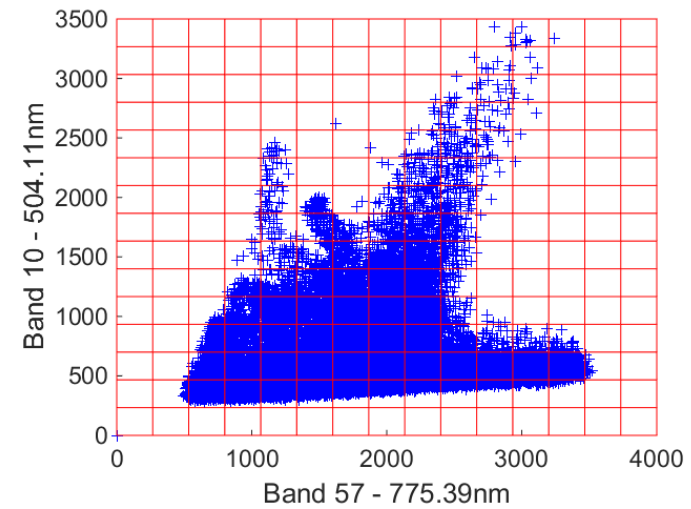

(a) Moll Harris data

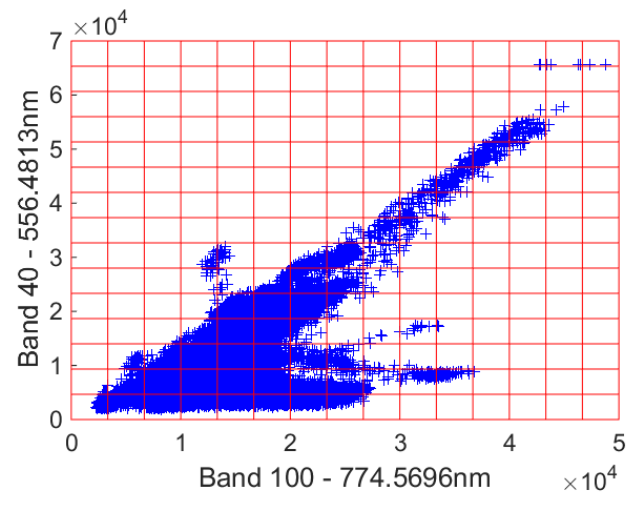

(b) Porton Down data

Figure 7: Scatter plot divided into cells

To find outliers, the ratio between the number of pixels in the foreground $\left(C_{F G}\right.$; blue zone $)$ and background $\left(C_{B G}\right.$; red zone) were examined (Figure 6$)$. This ratio is calculated for every pixel using Equation 5, where $\mathrm{N}$ is now the number of pixels in the cell.

$$
R=\frac{\sum_{n=1}^{N} C_{F G}}{\sum_{n=1}^{N} C_{B G}}
$$

\subsubsection{Band Selection}

Various methods were explored to create the scatter plot that the POHMT technique can be used on. The four best that have been explored so far are detailed below.

Random The first and most basic technique was to use two randomly selected bands to create the scatter plot. The idea behind this is that it gives a very quick way of detecting outliers in the dataset.

5 Random A variation of the random technique, is to do the random process 5 times. Cumulate the outliers found for all five, then discard all the outliers that only appear once. This method is slightly slower than the first but allows for more accurate outlier detection as more of the data is included in the calculations.

Principal Component Analysis (PCA) ${ }^{9}$ The next alternative approach for band selection that was looked at was to use the first two principal components to create the scatter plot. The POHMT technique is then performed on this scatter plot and the outliers are produced as normal. The idea behind this is that in the original method two randomly selected bands are used, therefore the majority of the data is discarded. By using the first two principal components, this scatter plot more accurately represents the entire dataset.

Normalized Difference Vegetation Index (NDVI) ${ }^{10}$ The final method that was explored was to use a variation of the NDVI. This is normally used to classify pixels that are vegetative and non-vegetative. This is done by looking at a band in the near-infrared (NIR) region, approximately 750nm, and a band in the visible region, approximately $650 \mathrm{~nm}$. These points in the spectrum are either side of the chlorophyll edge, or red edge, where there is a rapid change in reflectance of vegetation. Equation 6 is used to calculate the difference of the bands. An example of how this can be applied is shown in Figure 8.

$$
N D V I=\frac{N I R-V I S}{N I R+V I S}
$$




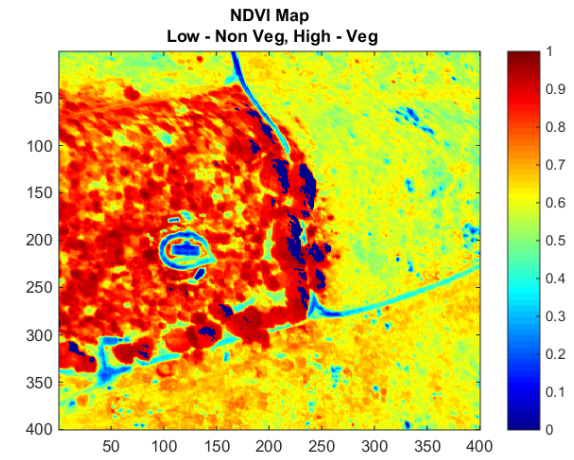

(a) Moll Harris data

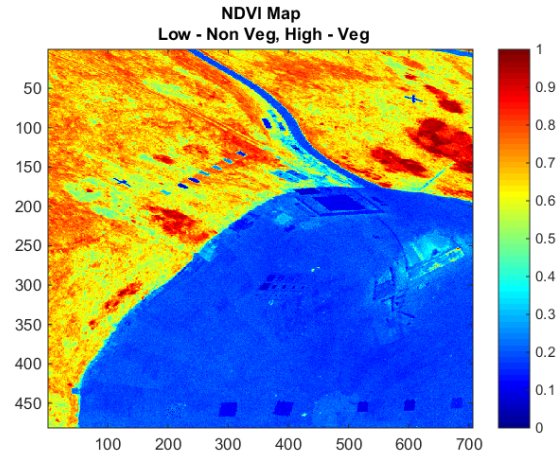

(b) Porton Down data

Figure 8: NDVI Plot

\section{RESULTS AND ANALYSIS}

To compare any algorithms produced against any existing techniques it was necessary to produce results for the SMACC algorithm. Before this could be performed the targets in each image had to be selected, see Figure 9.

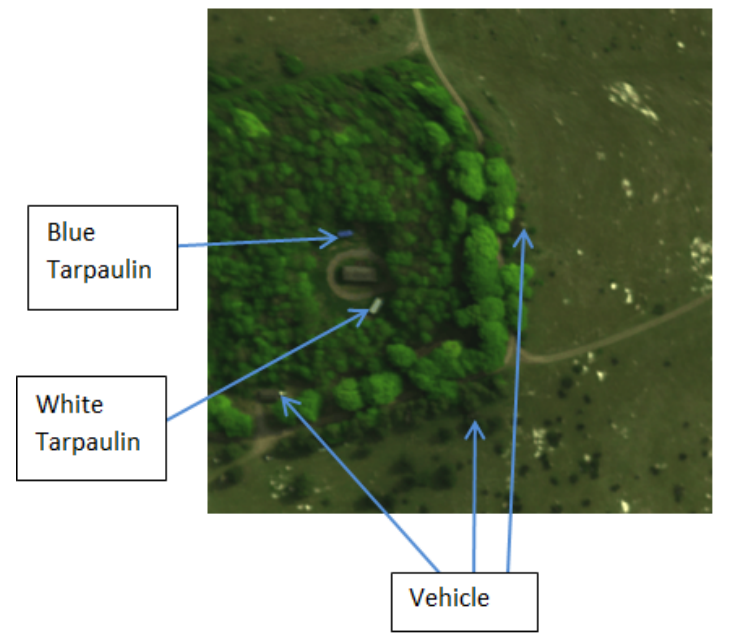

(a) Moll Harris data

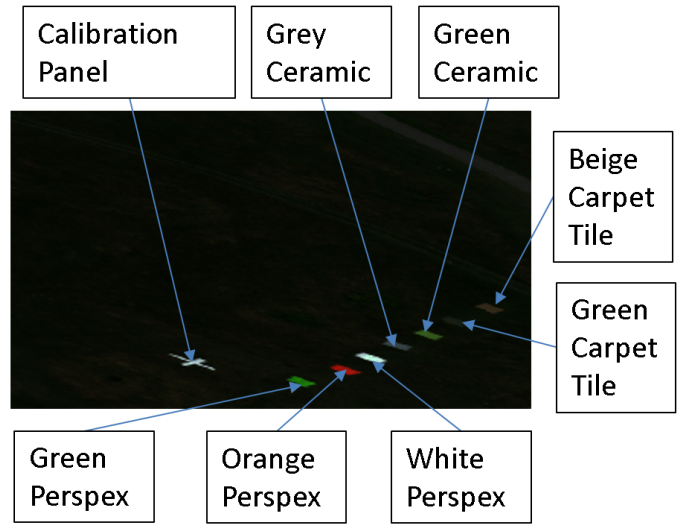

(b) Porton Down data

Figure 9: Target Locations

Both the SMACC and MD algorithms were then run on both dataset the results are shown in Figures 10 and 11.Also shown below are statistics for targets detected, targets missed and false positives for each algorithm, see Table 1. The results show that for the Moll Harris dataset the SMACC algorithm produces the best results as it detects 4 out of the 5 targets. For the Porton Down dataset the results are fairly similar and the SMACC algorithm is only slightly better as it produces fewer false positives. From this it is confirmed that there is indeed room for improvement on both these algorithms. 

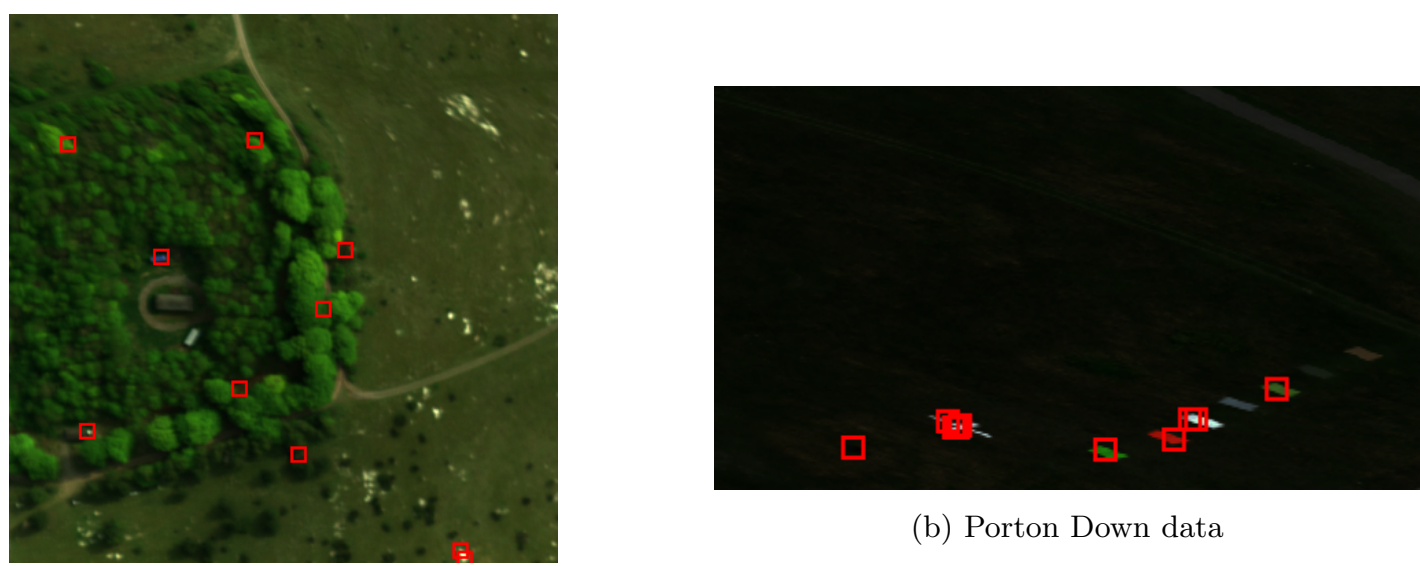

(a) Moll Harris data

Figure 10: SMACC Results
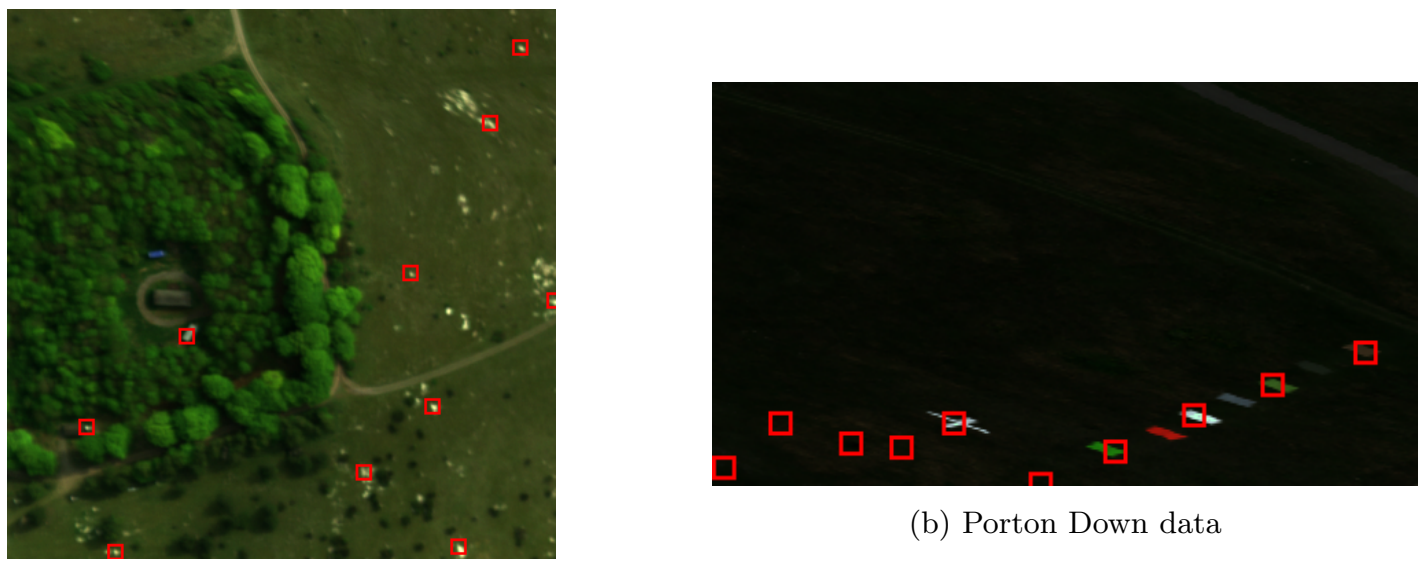

(b) Porton Down data

(a) Moll Harris data

Figure 11: Mahalanobis Results

\begin{tabular}{lccc} 
Dataset & Algorithm & Targets Detected & Targets Missed \\
\hline Moll Harris & SMACC & 4 & 1 \\
Moll Harris & MD & 2 & 3 \\
Porton Down & SMACC & 5 & 3 \\
Porton Down & MD & 5 & 3
\end{tabular}

Table 1: SMACC and MD Results for both datasets

As the second proposed algorithm has several variables that will each produce different results, in order to compare the methods, a ground truth for the image was created; see Figure 12. Each separate algorithm was then run on the image to produce a set of targets. This was then compared with the ground truth and the score was calculated by using Equation 7.

$$
\text { Score }=\frac{\text { Targets Found }}{\text { No. of Targets }}
$$




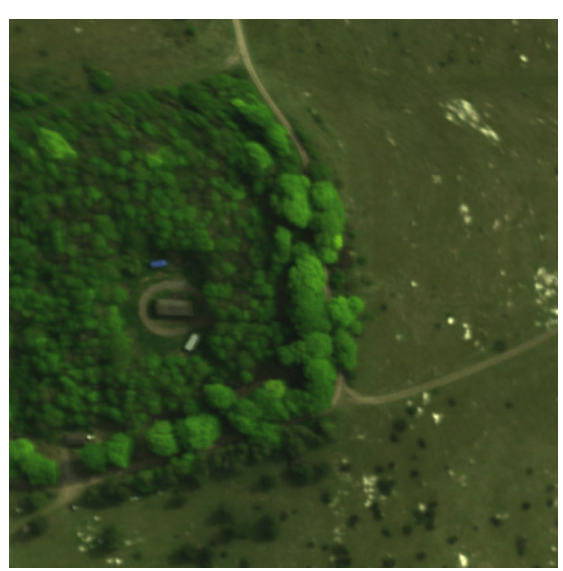

(a) Moll Harris data

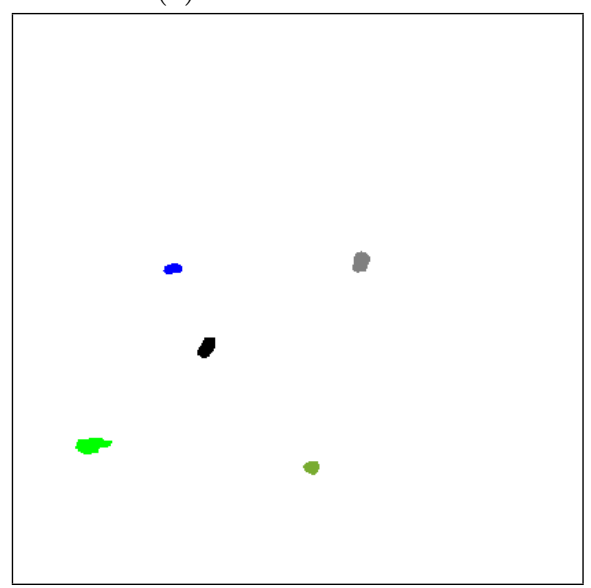

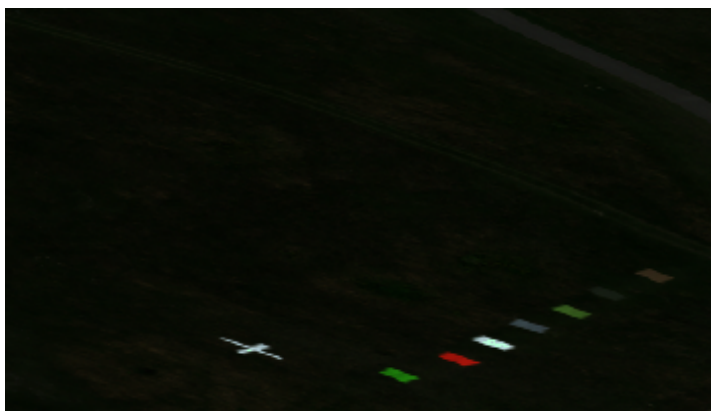

(b) Porton Down data

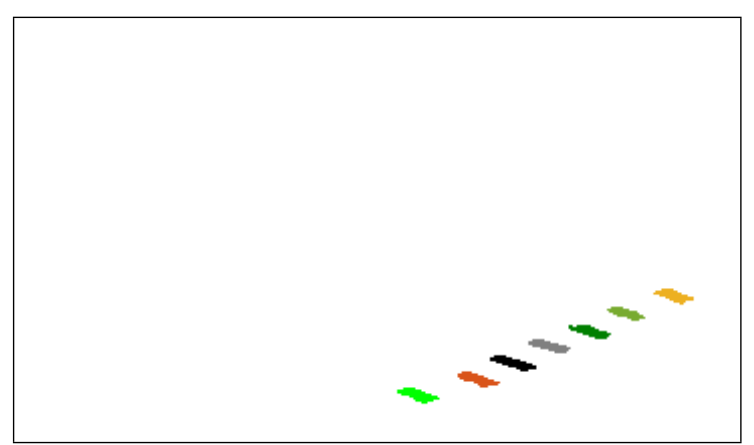

(d) Porton Down data

(c) Moll Harris data

Figure 12: Ground Truth

For every method a cell size from 5 to 150 was used going up in increments of 5 . As both the PCA and NDVI produce the same results every time, these were both run once and the results recorded. Due to the random nature of the Random and 5 Random techniques these were both run 1000 times and the average score for each cell size was recorded. The results are shown below, see Figure 13. Also shown are the two standard target detection approaches discussed earlier, the Sequential Maximum Angle Convex Cone and Mahalanobis Distance. 


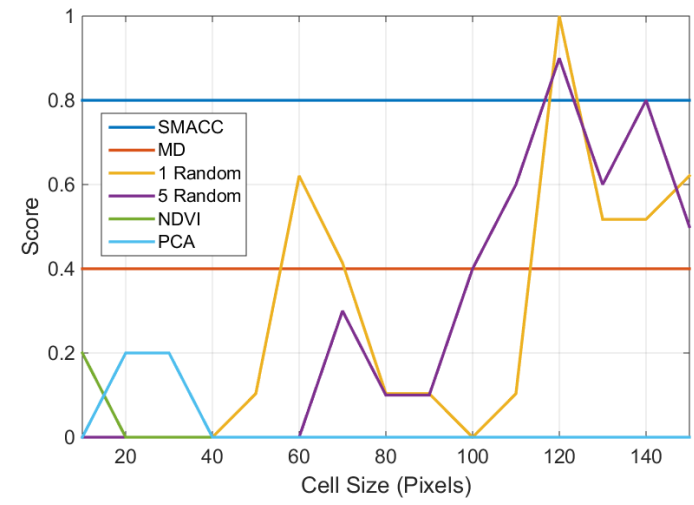

(a) Moll Harris data

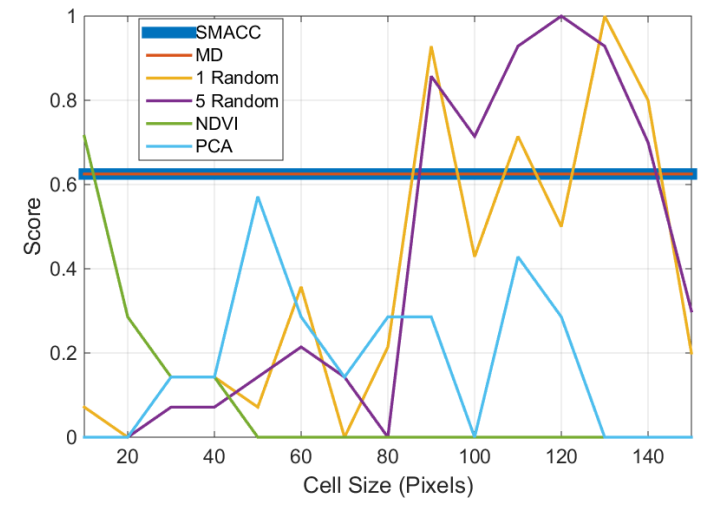

(b) Porton Down data

Figure 13: Results for each algorithm for every cell size

\section{SPEED TEST}

Although in some cases the POHMT will calculate the outliers faster than both the other techniques, due to the random nature of the method the time is not consistent like the other two, see Figure 14. For cell sizes between 20 and 50 the time for all techniques except the POHMT using the PCA bands is similar. When the cell size is increased beyond 70 pixels the time taken also increases quite drastically.

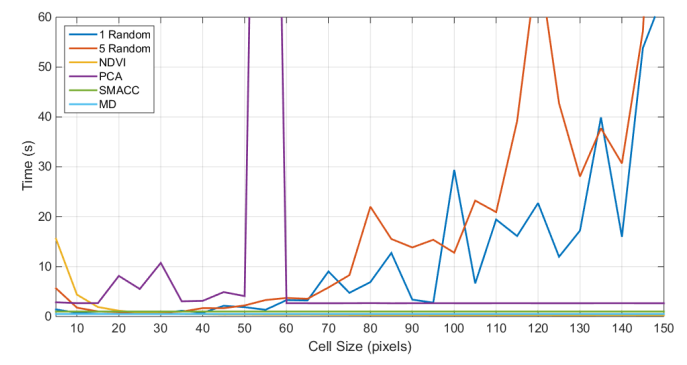

(a) Moll Harris data

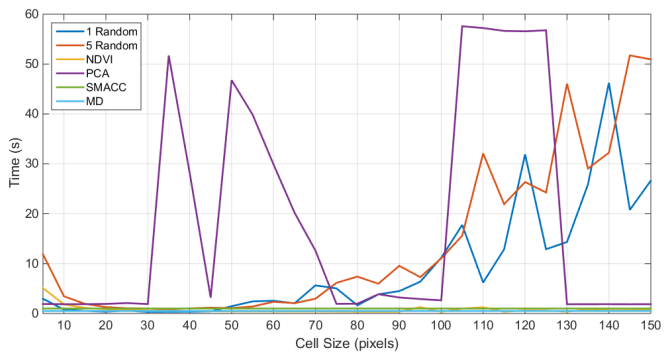

(b) Porton Down data

Figure 14: Speed Test for each algorithm on both datasets

\section{CONCLUSION}

The main object of the project was to produce an improved target detection method. Under certain circumstances this has been achieved using the POHMT with a pair of random bands selected, see Figure 13. However for the majority of cell sizes this method does not yield a better result. Further work will have to be carried out to see if the addition of multiple scatter plots or the use of selected pairs of bands, e.g. NDVI, PCA, can improve upon the results. Also when more datasets are available it will be necessary to repeat the analysis to validate the results.

\section{FUTURE WORK}

Future work will be carried out on examining ways to automatically define the size of cell to use when splitting up the scatter plot for use in the POHMT method. Also, the spectral unmixing is only one part of a two-stage process. Further research will be carried out to develop a technique based on the MODerate resolution atmospheric TRANsmission (MODTRAN) modelling code [5]. By estimating the important atmospheric parameters from data pertaining at the time of measurement, we plan to develop a robust atmospheric correction technique based on MODTRAN look-up tables. This may simplify the process of spectral unmixing to allow more accurate spectral matching and target detection. 


\section{ACKNOWLEDGEMENTS}

The authors would like to thank BAE Systems Military Air \& Information, the University Defence Research Collaboration (UDRC) and Defence Science and Technology Laboratory (DSTL) for providing the image data. This work was supported by an EPSRC-BAE Systems CASE studentship.

\section{REFERENCES}

[1] Shaw, G. A. and Burke, H.-h. K., "Spectral imaging for remote sensing," Lincoln Laboratory Journal 14(1), 3-28 (2003).

[2] Manolakis, D., Siracusa, C., and Shaw, G., "Hyperspectral subpixel target detection using the linear mixing model," Geoscience and Remote Sensing, IEEE Transactions on 39(7), 1392-1409 (2001).

[3] Nascimento, J. M. and Bioucas-Dias, J. M., "Nonlinear mixture model for hyperspectral unmixing," in [SPIE Europe Remote Sensing], 74770I-74770I, International Society for Optics and Photonics (2009).

[4] Gruninger, J. H., Ratkowski, A. J., and Hoke, M. L., "The sequential maximum angle convex cone (SMACC) endmember model," in [Defense and Security], 1-14, International Society for Optics and Photonics (2004).

[5] Smetek, T. E. and Bauer Jr, K. W., "A comparison of multivariate outlier detection methods for finding hyperspectral anomalies," Military Applications Society , 3 (2008).

[6] De Maesschalck, R., Jouan-Rimbaud, D., and Massart, D. L., "The Mahalanobis distance," Chemometrics and intelligent laboratory systems 50(1), 1-18 (2000).

[7] Raducanu, B. and Grana, M., "A grayscale hit-or-miss transform based on level sets," in [Image Processing, 2000. Proceedings. 2000 International Conference on], 2, 931-933, IEEE (2000).

[8] Murray, P. and Marshall, S., "A new design tool for feature extraction in noisy images based on grayscale hit-or-miss transforms," Image Processing, IEEE Transactions on 20(7), 1938-1948 (2011).

[9] Jolliffe, I., [Principal component analysis], Wiley Online Library (2002).

[10] Haboudane, D., Miller, J. R., Pattey, E., Zarco-Tejada, P. J., and Strachan, I. B., "Hyperspectral vegetation indices and novel algorithms for predicting green LAI of crop canopies: Modeling and validation in the context of precision agriculture," Remote sensing of environment 90(3), 337-352 (2004). 